\title{
Proceedings of the Annual Meeting of the British Neuropsychiatry Association, the Institute of Child Health, central London, 15-16 February 2001
}

\author{
Advances in brain stimulation \\ techniques
}

M GEORGE

Medical University of South Carolina, USA

Aims: To critically review the exciting recent advances in new, minimally invasive brain stimulation (MIBS) techniques. Particular attention will focus on their potential antidepressant effects, ability to be coupled with functional brain imaging techniques, and known neurobiological mechanisms of action.

Methods: The current MIBS renaissance builds on recent knowledge from the field of functional brain imaging, as well as classic teachings from neuropsychiatry. The past decade has seen fundamental advances in the following MIBS techniques: transcranial magnetic stimulation (TMS), vagus nerve stimulation (VNS), conventional electroconvulsive therapy (ECT) (now modified and produced by magnetic currents: (magnetic seizure therapy (MST)), and finally, deep brain stimulation (DBS). Antidepressant clinical trial data will be reviewed for each technique, as well as data from their combination with functional brain imaging.

Conclusions: These tools hold the promise of a new age for neuropsychiatry, from both a research and clinical perspective. As research tools, MIBS techniques can formally test hypotheses about brain-behaviour relations. Although still crude clinically, several of the techniques have shown early promise, particularly in the area of depression. Unfortunately, rapid clinical progress is hampered by incomplete understanding of the effects of brain stimulation at a cellular, neurotransmitter, or genetic level, particularly as function of stimulation use parameters (frequency, intensity, pulse width, duty cycle, and dose) Nevertheless, these advances suggest that perhaps the real golden age of neuropsychiatry was not during the time of Ferrier, Jackson, and Gowers. Rather, armed with advanced brain scans and MIBS tools (and full knowledge of their neurobiological effects), neuropsychiatrists of the near future will be able to use MIBS techniques to research and probe the normal human brain and then to correct its dysfunctions.

Transcranial magnetic stimulation of the brain: mechanisms and application

JC ROTHWELL

Institute of Neurology, London, UK

Aims: To understand more about the physiological mechanism(s) responsible for long term effects of repetitive transcranial magnetic stimulation (rTMS) on the human cerebral cortex.

Methods: These experiments use the motor cortex as a model for the effects of rTMS on cortical mechanisms. This is because the effects of TMS on the motor cortex can easily be quantified by measuring the size of the twitches that stimulation produces in contralateral muscles. The general design is to use single or paired pulse stimulation to test the excitability of the motor cortex before and after a period of rTMS. The data can then be interpreted in terms of changes in neuronal excitability or synaptic efficacy. Different durations of rTMS were given to the motor and/or premotor cortex of 10 normal volunteers and the effect on motor cortex excitability evaluated up to 1 hour afterwards. In these experiments rTMS was always given at a frequency of $1 \mathrm{~Hz}$ and at an intensity that was $80 \%-90 \%$ of the threshold needed to provoke a muscle twitch in voluntarily activated muscle.

Results: rTMS of the motor cortex reduced the excitability to subsequent test pulses. The duration of the after effect was related to the number of stimuli in the rTMS train. 1500 stimuli produced an effect that lasted 20 minutes, whereas the effect of 300 stimuli lasted 5 minutes or less. The size of the after effect did not depend so clearly on the number of rTMS stimuli. Stimulation over the premotor cortex also produced changes in the excitability of the motor cortex, and these had a different threshold to those produced by direct stimulation of the motor cortex itself. Stimulation over either motor or premotor cortex produced behavioural effects: long latency transcortical stretch reflexes were reduced and reaction times to visual stimuli were prolonged. These effects lasted up to 15 minutes after 1500 stimuli. Conclusions: Repetitive TMS of motor and/or premotor cortex affects the excitability of intrinsic cortical mechanisms for several minutes after the end of stimulation. This is paralleled by changes in motor performance. Finally, stimulation of one area of cortex (premotor) can affect processing in an adjacent, connected area of cortex, suggesting that the actions of rTMS can spread away from the site of stimulation and change the way data are processed within functional circuits of the cortex.

\section{Neural transplants for}

neurodegenerative diseases: a fading dream?

R BARKER

Cambridge Centre for Brain Repair and Addenbrooke's NHS Trust, Cambridge, UK

Neurodegenerative disorders comprise a group of diseases in which there is a loss of defined populations of neurons within the central nervous system leading to progressive neurological disease. This loss can occur through genetic causes as in Huntington's disease or through less well defined processes such as idiopathic Parkinson's disease. In all cases the loss of neurons leads to the development of a range of clinical features many of which respond to symptomatic drug treatment but none of which are amenable to cure. In this talk I shall begin by reviewing the experimental evidence that neural transplantation is curative in animal models of Parkinson's and Huntington's disease. This will lead on to a summary of the current status on neural grafting clinically with respect to Parkinson's and Huntington's disease, and will conclude with a discussion on the future of neural transplantation as a means of effecting brain repair.

\section{Organisation of the visual brain}

$S$ ZEKI

Wellcome Department of Cognitive Neurology, University College London, UK

A great deal has been learned about the organisation of the primate visual brain in the past 25 years. In particular, it has been shown that the visual brain consists of many specialised visual areas which undertake different tasks. The further demonstration that activity in different areas reach a perceptual end point at different times, suggests that these areas are fairly autonomous from one another. This latter suggestion is strongly supported by the clinical evidence which also shows that activity in individual areas can lead to conscious perception of the attribute for which that area is specialised without even the participation of the primary visual correct, area V1.

Functional neuropathology of "positive" visual perceptual disorders

D FFYTCHE

Institute of Psychiatry, London, UK

In 1942, Heinrich Klüver's experiments with mescaline led him to conclude that the visual illusions and hallucinations of psychotomimetic drugs, cerebral lesions, migraine, eye disease, and hypnagogia were identical, falling into a limited range of distinct categories. He reasoned that each category revealed a fundamental visual mechanism but predicted that an understanding of their neurobiology would await future research developments. The organisational principle of functional specialisation and the advent of non-invasive cerebral imaging have provided the theoretical background and investigative tools to test Klüver s predictions. Using fMRI, we have characterised the neural activity associated with different categories of visual hallucination and have found a simple relation between visual brain and visual mind: increased activity within cortex specialised for a given visual attribute results in a hallucination of that attribute. While the 
underlying cytoarchitecture and physiology of the activity has yet to be determined, it is likely to involve subsets of neurons whose activity correlates with consciousness. Our phenomenological survey adds support to our conclusions by revealing clinical associations between hallucination categories, which reflect anatomical associations between functionally specialised cortical areas. The picture that emerges is of a range of "opositive" perceptual pathologies, each related to increased activity within a different specialised cortical area. The functional pathology causing the increased activity varies between clinical conditions; however, its perceptual outcome remains the same. The findings help explain Klüver's observations and point to important but, as yet, unidentified specialisations in the human visual system.

\section{Visual hallucinations in dementia}

C BALLARD

Newcastle General Hospital, Newcastle, UK

Aims: To examine the association between visual impairment and visual hallucinations in people with dementia

Methods: Three cohorts were studied, (a) people meeting NINCDS ADRDA criteria for probable Alzheimer's disease $(n=50),(b)$ a clinical cohort meeting NINCDS AIRENS criteria for probable or possible vascular dementia ( $\mathrm{n}=92),(c)$ people meeting international consensus criteria for dementia with Lewy bodies $(n=36)$. All three groups had standardised evaluation of visual hallucinations. Visual accuity was assessed in Cohort $\mathrm{A}$, and patients received a detailed examination for eye pathology. Cohorts B (CAMCOG) and C (cognitive research battery) were evaluated using standardised neuropsychological instruments.

Results: In cohort A, there was a strongly significant relation between impairment of visual acuity and the presence of visual hallucinations. No patients with normal acuity experienced hallucinations in the visual modality. Of the specific eye pathologies, only cataracts were associated with visual hallucinations. In cohort B there was a significant association between visual hallucinations and visual agnosia, whereas in cohort $\mathrm{C}$ a significant relation was identified between visual hallucinations and impairment of visual attention.

Conclusions: Impairments in visual processing at various levels are associated with visual hallucinations, with mechanistic implications. The role of treating eye pathology in the management of visual hallucinations needs to be explored.

\section{Visual agnosias and related disorders}

T LANDIS

HCUC, Geneva

Visual disorders can be viewed as resulting from damage to bottom up processing, when early visual properties such as the perception of form (appeceptive visual agnosia), colour (achromatopsia), or motion (akinetopsia) are damaged. However, most classic visual agnosias, such as visual associative agnosia, prosopagnosia, pure alexia, and colour agnosias are the consequence of unilateral cerebral damage, leaving intact the visual processing of the non-damaged hemisphere.
Primary visual information is thus perceived but not necessarily conveyed to the other hemisphere. Such visual agnosias can be viewed as disconnection syndromes, implying that there are two visual systems differently specialised. This specialisation extends also to emotional information.

\section{Migraine auras damage the visual pathways}

NJ LEGG, N KHALIL

Hammersmith Hospital, London, UK

Aims: To assess visual function in migraine patients by psychophysical and physiological tests.

Methods: 92 patients and 62 controls were studied. Psychophysical tests included spatial and temporal contrast sensitiveity (CS) and perception of illusions. Physiological tests comprised electroretinogram and visual evoked responses (VEP) to single flash, repetitive flash, and pattern reversal (PR). Results: In three of our tests abnormal results were found to be correlated with duration of migraine. These were spatial CS, temporal CS, and P100 amplitude in the PRVEP. These results were confined to migraine with aura (MA), and they were not age effects. P100 amplitudes were reduced by $36 \%$ in subjects with MA of more than thirty years duration compared with durations of less than 10 years. In patients with a consistently lateralised aura hemifield testing showed that the decline in spatial CS and P100 amplitude was confined to the side of the aura.

Conclusions: Repeated migraine auras cause damage to the visual system, probably at cortical level, and predominantly to the M pathway. The pathophysiology is likely to be ischaemia, and possibly NMDA neurotoxicity, during the aura itself.

\section{Abnormal colour after images in Alzheimer's disease: a defect of primary visual cortex}

D CHAN, S CRUTCH, E WARRINGTON

The National Hospital for Neurology and Neurosurgery, London, UK

Aims: To investigate the abnormal colour after-images experienced by a patient with Alzheimer's disease (AD) affecting the posterior cerebral cortex.

Methods: A 64 year old woman had a clinical diagnosis of posterior cortical atrophy due to AD. Her subjective experience of abnormally intense and prolonged colour after-images was assessed further using psychophysical tests including tests of primary visual functions and tests of colour after image generation.

Results: She exhibited minor impairment of some aspects of primary visual function, with partial impairment of colour vision but preserved colour constancy. She was found to experience consistently colour after-images of abnormal latency, duration, and amplitude. Monocular testing resulted in the perception of colour after-images through the unexposed eye. Retinal function was normal. Conclusions: Test results indicated that colour after-images originate in the primary visual cortex (V1) rather than the retina or cortical area V4. A mechanism for the generation of colour after-images is proposed which involves rebound inhibition of adapted wavelength-selective V1 neurons. Her pres- entation is considered to represent excessive rebound inhibition of previously stimulated V1 neurons, as a consequence of the relative sparing of interneurons in V1 in the context of $\mathrm{AD}$ related degeneration of excitatory $\mathrm{V} 1$ neurons.

\section{Coloured filters and their clinical benefits}

A WILKINS, N SIHRA

University of Essex, Colchester, UK

Aims: To assess the claims concerning benefit from coloured filters

Background: We have shown that reading is impaired by visual stress in $20 \%$ of unselected schoolchildren, substantially so in $5 \%$. We have developed a new method for treating visual stress and improving reading fluency using coloured filters, either sheets of coloured plastic placed over a page of text when reading, or coloured lenses worn as glasses.

Methods: We examined participants who had worn tinted glasses for 3 months to 4 years (average 2 years) and who reported benefits. They read aloud different passages of randomly ordered common words repeatedly, and did so in two sessions, spaced about 2 weeks apart. The reading speed was measured under light of different colours. A 2 D surface was then fit to the data using least squares to describe the change in reading rate as a function of chromaticity.

Results: The 2 D surfaces accounted for an average across participants of $30 \%$ of the variance and showed consistency within participants from one test session to the next, but with large differences between participants. There was no tendency for colours complementary to those at which reading speed was maximum to show a slower than average speed; rather speed was usually minimal when saturation was weak. The functions suggest that departures from optimal chromaticity of a few just noticeable differences (jnds) can have a large effect on reading speed. This specificity of therapeutic colour has been used as the basis of two double masked studies in which clinical benefits of tinted lenses have been shown in children with reading difficulties and adults with migraine.

Conclusion: For optimal clinical effect the chromaticity of an ophthalmic tint must be selected with precision; the tint thus chosen is reliable. Opponent colour mechanisms are not involved.

\section{The neural mechanisms of visual} conversion disorder: a functional magnetic resonance imaging study

DJ WERRING, L WESTON, ET BULLMORE, GT PLANT, MA RON

Institute of Neurology, London, UK

Aims: The neural mechanisms underlying visual conversion symptoms are not well understood, but may be investigated using functional imaging. This study used functional magnetic resonance imaging (fMRI) to investigate the cerebral response to visual stimulation in patients with visual conversion disorder in comparison with healthy individuals.

Methods: fMRI was used to detect brain activation induced by periodic monocular $8 \mathrm{~Hz}$ photic stimulation in five patients with asymmetric visual loss for which no organic cause 
could be identified despite extensive investigation; and in seven controls.

Results: Patients showed a reduced power of response to visual stimulation in bilateral areas of visual cortex, but relatively increased activity in bilateral inferior frontal cortex, left insula, claustrum and striatum, bilateral thalami, and left posterior cingulate cortex.

Conclusions: These results demonstrate an abnormal cerebral response to simple visual stimulation in patients with visual conversion disorder compared with healthy controls. The regions showing increased response have been implicated in hypnotic states and the attentional modulation of responses in visual cortex. Furthermore, activation of this network may reflect a shift towards nonconscious (implicit) processing of visual stimuli. However, the variation in responses in individual patients emphasises the complexity and heterogeneity of the neural correlates of visual conversion disorder.

\section{Effects of dosage insufficiency of $\mathbf{X}$ linked genes on brain structures and visual processing style}

J KUNTSI, K ELGAR, C GOOD, D SKUSE Institute of Child Health, Lodon, UK

Aims: We aimed to investigate the effects of dosage insufficiency of $\mathrm{X}$ linked genes on both brain structures and specific cognitive abilities. Our subjects had a single X chromosome $(45, \mathrm{X})$, and a Turner syndrome phenotype, which is associated with normal verbal intelligence but significant deficits in visuospatial and face processing.

Methods: The cognitive study involved assessing 23 45,X women and 23 matched controls. We undertook structural MRI and voxel based morphometry, as well as focused tests of visuospatial and face processing abilities. Our measures included novel assessments, with an emphasis on feature based compared with configural visual processing style.

Results: The cognitive data supported our hypothesis that $45, \mathrm{X}$ women would be more likely than controls to process complex visual information in a feature based manner. For example, they commenced a copy of the Taylor figure in a feature-based fashion $(p=0.006)$ and they identified relatively fewer photographs of famous faces when featural information was removed $(p=0.04 ; p=0.02$ for RT data).

Their face processing deficits do not simply reflect memory related problems for visual stimuli: 45,X women also perform poorly on tasks involving simultaneous $(\mathrm{p}<0.001)$ and immediate $(\mathrm{p}=0.005)$ face matching. There were substantial but specific deficits in emotion recognition too, in particular in the recognition of fear and anger $(p<.001)$. The brain imaging data showed that a $45, \mathrm{X}$ karyotype was associated with increases in grey matter in the amygdala $(\mathrm{R}>\mathrm{L})$ and orbitofrontal cortex (bilateral), and decreases in the occipitoparietal ( $\mathrm{R})$ cortex.

Conclusions: The data indicate that $\mathrm{X}$-monosomy is associated with a specific cognitive and brain phenotype. These findings give insight into neurocognitive systems that are affected by dosage insufficiency of $\mathrm{X}$ linked genes. Our ongoing investigations of Turner syndrome women with partial deletions of the $\mathrm{X}$ chromosome aim to identify the genes responsible.

\section{Anxiety episodes after anterior-temporal lobectomy. Panic attacks or ictal panic?}

MV LAMBERT, CD BINNIE, AS DAVID

Institute of Psychiatry, London, UK

Aims: To differentiate between panic attacks and ictal panic in a patient with epilepsy Method: Detailed review of clinical history along with EEG and neuroimaging investigations

Results: A set of guidelines to help differentiate the two conditions

Conclusions: Panic attacks and ictal panic can be difficult to differentiate in a person with epilepsy. Guidelines can help to determine the correct diagnosis and thus the correct treatment.

\section{Illness awareness and neuropsychological functioning in first onset psychosis}

KD MORGAN, PD DAZZAN, C MORGAN, M SHARPLEY, R MALLET, A DAVID, J LEFF, RM MURRAY Institute of Psychiatry, London, UK

Aims: In psychosis, there is some evidence that reduced general cognition and decreased frontal lobe functioning are associated with poor illness awareness and the ability to relabel symptoms as psychotic. This study investigated this further in a group of 103 first onset psychosis patients (mean age 29, 62 males, DSM-1V; schizophrenia=60, "other psychoses"=43).

Methods: Patients were assessed with David's insight schedule and a neuropsychological test battery rating IQ, executive function, and verbal fluency.

Results: Patients with poor illness awareness scored lower on performance IQ (ANOVA, $\mathrm{p}=0.011$ ) and trail making A (ANOVA, $\mathrm{p}=0.020$ ), compared with high awareness patients. A linear regression, controlling for age, sex, ethnicity, diagnosis, and years of education, showed that only trail making A scores were associated with awareness $\left(R^{2}=0.080, \mathrm{p}=0.004\right)$. Patients less able to relabel symptoms as psychotic scored lower on performance IQ (ANOVA, $\mathrm{p}=0.031$ ) compared with patients showing good relabelling. A linear regression showed lower performance IQ, being older and being male were associated with reduced ability to relabel $\left(R^{2}=0.181, \mathrm{p}<0.001\right)$.

Conclusion: These findings indicate that tasks relatively independent of language have more impact on illness awareness. The mental fluidity necessary in performance IQ and trail making may correspond with the capacity to self appraise one's psychotic status and might also indicate that this capacity may be most dependent on right hemispheric brain function.

Insight, social knowledge, and working memory in schizophrenia

R UPTHEGROVE, F OYEBODE

Solihull Healthcare NHS Trust, UK

Aims: How insight and social knowledge affect outcome in schizophrenia is an important question. Few studies have looked at working memory in this context. It was the aim of this study to look at the relation between insight, social knowledge, and work- ing memory in the context of Detention under the Mental Health Act.

Method: Insight rating scale (SAI-E), social knowledge questionnaire (SKQ), and working memory tests were administered to a group of patients with schizophrenia detained under the Mental Health Act 1983. The results were compared with that of a control group of informal inpatients with schizophrenia.

Results: Detained patients scored significantly worse on the insight $(\mathrm{p}=0.003)$ and social knowledge $(p=0.007)$ rating scales, yet there was no positive correlation between the two deficits. Impaired insight was independent of severity of illness as measured by the BPRS. Results for working memory were inconclusive.

Conclusion: Poor insight and impaired social knowledge were significantly, but independently, associated with detention under the mental health act. This suggests that insight and social knowledge are distinct skills. Working memory may have a role in determining the ability to hold information, weigh it in the balance, and reach a decision. How this relates to insight and social knowledge warrants further investigation.

\section{Effects of psychological therapy on \\ brain activation in patients with a diagnosis of schizophrenia}

J MELLERS, T WYKES, M BRAMMER Maudsley Hospital, London, UK

Aims: Changing the cognitive deficits of patients with schizophrenia has become a focus of attention in recent years as evidence has emerged on the specific effectiveness of both psychological and pharmacological therapies. The results of one randomised control trial testing a psychological rehabilitation programme aimed at information processing strategies showed improvements in cognition post-treatment. The current paper concentrates on whether there are concomitant brain activation changes, in addition to the previously reported behaviour changes, as a result of engaging in cognitive remediation therapy (CRT).

Methods: Three groups, patients receiving control therapy or CRT and a normal control group, were investigated in a repeated measures design using a working memory paradigm (the 2 back test). Functional MRI data were collected during the task and a broad assessment of executive functioning was completed at baseline and post-treatment outside the scanner. Brain activation changes were identified after accounting for possible task correlated motion artefact.

Results: The fMRI analyses indicate that there are changes over time in the patient groups, which differ from those in the control group. The control group reduced activation but the two patient groups showed in all cases an increase in activation over time. The patient group who received successful cognitive remediation therapy significantly increased their brain activation in regions, particularly frontocortical areas, associated with working memory

Conclusions: This is the first time that brain activation changes in a seriously disabled group of patients with schizophrenia can be clearly associated with psychological rather than pharmacological therapy. These data can now be added to the growing corpus of findings of psychological interventions having a direct effect on brain functioning. 


\section{Memory impairment evaluation in incipient Alzheimer's disease}

A IVANOIU, S ADAM, M VAN DER LINDEN, AC JUILLERAT, A JACQUEMIN, G GODFRIND, C PRAIRIAL, R MULLIGAN, M GEORGE, S BECHET, E SALMON, $\mathrm{X}$ SERON

University of Aberdeen, Aberdeen, UK

Aims: To evaluate the usefulness of a new episodic memory test for the diagnosis of Alzheimer's disease (AD) in its very incipient stage.

Methods: A delayed recall test using semantic cues ( $S$ Adam, $M$ Van der Linden, not published) was compared with three well known episodic memory tests. Seventy one outpatients consulting a memory clinic were included. All underwent brain imaging and were reexamined 1 year later to confirm the initial diagnosis. Twenty four showed clear memory impairment but were not demented at the first examination (incipient AD), 26 had mild $\mathrm{AD}$ and 21 had subjective memory complaints. They were also compared with 38 normal elderly people.

Results: No difference was seen between subjective complainers and normal aged. Incipient $\mathrm{AD}$ patients were impaired on all memory tests. However, an analysis of the sensitivity/ specificity showed the cued recall test is the most discriminant measure $(89 \% / 94 \%$, by the ROC curve).

Conclusions: The cued recall seems to be a sensitive marker for incipient predemential AD.

\section{A comparison of handedness in preterm born and full term born adults}

M ROONEY, M ALLIN, L RIFKIN, J TOWNSEND, AL STEWART, M HOTOPF, RM MURRAY Institute of Psychiatry, London, UK

Aims: To see if there are higher rates of left or mixed handedness in preterm born adults compared with full term born adults, as a result of compensation for early brain insults. Methods: We administered the Edinburgh handedness inventory to a cohort of adults born in 1979-81, before 33 weeks gestation in University College Hospital London. This is a questionnaire that elicits laterality for different tasks. More than half this cohort were previously shown to have brain abnormalities when assessed by MRI scan, most commonly of the ventricles, the corpus callosum, and white matter. We also administered the inventory to a group of controls of the same age

The subjects were 114 preterm born people aged 17.5 to 18.5 (66.3\% of the original cohort) and 69 full term born controls.

Results: The Kruskal-Wallis Test was used to compare scores on the Edinburgh handedness inventory. There was no significant difference between the two groups $\left(r^{2}=0.22\right.$, $\mathrm{df}=1, \mathrm{p}>0.05$ )

Conclusions: We found no difference in laterality between preterm and full term born individuals.

\section{Pathologies of awareness, neglected} issues

P HALLIGAN

Cardiff University, Cardiff, UK

Much of what we know about visual neglect has been inferred from research carried out at the functional or subpersonal level of the patient's performance. Disorders of neglect have been studied primarily within a functional information processing framework that has largely focused on disorders to unconscious mechanisms of "attention". Consequently, most studies have been concerned with what the patient can do despite or without phenomenological awareness. It is perhaps odd that neglect (the most common of attentional disorders) has attracted comparatively little interest from the standpoint of the patient's visual awareness by comparison with less common cases of "blind sight" and visual agnosia. As a result, some of the most interesting and common clinical findings that have long characterised the condition have remained mysterious and neglected. Informally, most students of neglect think of the condition as a disorder of visual awareness but with limited exceptions, this eminently reasonable belief has not been adequately investigated. In this talk, clinical examples from visual neglect will be used to demonstrate how consideration of the patient's explicit knowledge (that which can be expressed by the subject) together with more implicit forms of knowledge (traditionally revealed in task performance without the subject conscious awareness) can help to provide a more comprehensive explanation of the disorder and the normal functional systems involved.

\section{Insight and awareness of illness in neuropsychiatry}

A DAVID

Institute of Psychiatry, London, UK

Aims: The aim of this lecture is to compare and contrast the concepts of insight as applied to "psychiatric" and "neurological" disorders. This will include an exploration of whether lack of insight can be considered a neuropsychological deficit (anosognosia).

Methods: Historical review with illustrations from the research literature. Alzheimer's disease, focal neurological disorders, and schizophrenia will be discussed. In addition, empirical data on neuropsychological testing in patients with psychosis from various cohort studies and from patients with unexplained medical symptoms will be presented. Finally, recent functional neuroimaging data will be presented which examines the neural correlates of visual self recognition

Results: The data will show a rather weak contribution of cognitive impairment to awareness of illness in schizophrenia. A link between mood and awareness is shown in studies of Alzheimer patients and those with unexplained symptoms. A distinct neural network seems to underlie self-recognition in normal subjects.

Conclusions: Insight and awareness are highly amenable to empirical research and remain a crucial area in neuropsychiatry. Lack of awareness of disease requires an proper understanding of both general psychological and neuropsychological mechanisms. 